\title{
Ansätze for Self-Dual Yang-Mills Fields
}

\author{
R.S. Ward \\ Department of Mathematics, Trinity College, Dublin 2, Ireland
}

\begin{abstract}
A sequence $\mathscr{A}_{1}, \mathscr{A}_{2}, \ldots$ of ansätze for generating self-dual solutions of the Yang-Mills equations is presented. For each $n, \mathscr{A}_{n}$ produces a solution depending on two arbitrary functions of three variables. As an application, we see that $\mathscr{A}_{2}$ generates a static Yang-Mills-Higgs 2-monopole solution.
\end{abstract}

\section{Introduction}

In recent years, there has been considerable interest in self-dual $S U(2)$ YangMills fields in Euclidean space $\mathbb{R}^{4}$. In the first place, they arise as instantons, which dominate the Euclidean functional integral [1-3]. Secondly, they include, as a special case, static Yang-Mills-Higgs fields in space-time, in the Prasad-Sommerfield limit; these have come to be known as multi-monopoles [4-8]. One of the more successful ways of understanding the self-duality equation, and of generating solutions to it, has been the approach which arises out of Penrose's twistor theory [9]. This led to a sequence $\mathscr{A}_{1}, \mathscr{A}_{2}, \ldots$ of ansätze which generate all instanton solutions [10,11]; and led also the Atiyah-Hitchin-Drinfeld-Manin (AHDM) construction [3] which generates the instantons even more effectively. More recently, these ansätze have been used to construct multi-monopole solutions of the Yang-Mills-Higgs-Bogomolny (YMHB) equations $[7,8]$.

The purpose of this paper is twofold. First, a generalization of the ansätze $\mathscr{A}_{n}$ is described. These new $\mathscr{A}_{n}$ generate, for each $n \geqq 1$, a family of solutions of the self-duality equations depending on two free functions of three variables each. After a general description of the "twistor" construction in Sect. 2, the new $\mathscr{A}_{n}$ are presented in Sect. 3. There is also some discussion of the problem of how to ensure that a gauge field generated by $\mathscr{A}_{n}$ is smooth and real-valued, i.e. taking values in the Lie algebra of $S U(2)$ rather than that of $S L(2, \mathbb{C})$.

Section 4 brings us to the second topic of the paper. The multi-monopole solutions referred to above are all superimposed, axially-symmetric configu- 
rations. However, it was recently announced that the ansatz $\mathscr{A}_{2}$ can also be used to construct a non-axially-symmetric solution representing two separated monopoles [12]. Some details of this construction are presented here.

The discussion is essentially self-contained, but builds on the results of references [11] and [7].

\section{The General Construction}

Let $x^{\mu}$ be the standard coordinates on $\mathbb{R}^{4}$, and define new coordinates $x^{P Q}$ $(P, Q=1,2)$ by

$$
\left[\begin{array}{ll}
x^{11} & x^{12} \\
x^{21} & x^{22}
\end{array}\right]=\left[\begin{array}{cc}
x^{0}-i x^{3} & -i x^{1}-x^{2} \\
-i x^{1}+x^{2} & x^{0}+i x^{3}
\end{array}\right]
$$

Let $Z^{\alpha}=\left(\omega^{P}, \pi_{Q}\right)$ be four complex coordinates on "twistor space" $\mathbb{C}^{4}$ (i.e. $Z^{0}$ $\left.=\omega^{1}, Z^{1}=\omega^{2}, Z^{2}=\pi_{1}, Z^{3}=\pi_{2}\right)$. The basic equation expressing the relationship between $Z^{\alpha}$ and $x^{\mu}$ is

$$
\omega^{P}=x^{P Q} \pi_{Q} .
$$

The "reality" structure on $Z^{\alpha}$ is given by the antilinear map

$$
\begin{aligned}
Z^{\alpha} \mapsto Z^{* \alpha} & =\left(\overline{Z^{1}},-\overline{Z^{0}}, \overline{Z^{3}},-\overline{Z^{2}}\right), \\
\omega^{P} \mapsto \omega^{* P} & =\left(\overline{\omega^{2}},-\overline{\omega^{1}}\right), \\
\pi_{P} \mapsto \pi_{P}^{*} & =\left(\overline{\pi^{2}},-\overline{\pi^{1}}\right) .
\end{aligned}
$$

Equation (1) is preserved under this map, in the sense that $\omega^{P}=x^{P Q} \pi_{Q}$ if and only if $\omega^{* P}=x^{P Q} \pi_{Q}^{*}$. [This holds providing, of course, that $x^{\mu}$ is real.]

Let us assume from now on that $\pi_{1}$ and $\pi_{2}$ are not both zero (i.e. remove from $\mathbb{C}^{4}$ the subspace $\left.\pi_{P}=0\right)$. Then if we factor the $\pi_{P}$-space by the proportionality relation $\pi_{P} \sim \lambda \pi_{P}$ ( $\lambda$ being a non-zero complex constant), we obtain the complex projective space $\mathbb{C I P}^{1}$, which is simply the Riemann sphere. The antilinear map (2) is exactly the antipodal map on this sphere. In terms of the coordinate $\zeta=\pi_{1} / \pi_{2}$ (which is allowed to take the value $\infty$ ), the map (2) is $\zeta \mapsto$ $-\bar{\zeta}-1$.

Cover $\mathbb{C I P}^{1}$ with two patches $U$ and $\hat{U}$, such that $U$ contains the point $\zeta=0, \hat{U}$ contains the point $\zeta=\infty, U \cap \hat{U}$ is an annular region containing the circle $|\zeta|=1$, and $U$ and $\hat{U}$ are conjugate to each other (in the sense that the antipodal map (2) is a $1-1$ map of $U$ onto $\hat{U}$ ).

Now we are in a position to describe the construction. Let $g\left(Z^{\alpha}\right)$ be a 2-by2 matrix of functions of $Z^{\alpha}$, such that

(i) $g$ is homogeneous of degree zero in $Z^{\alpha}$, i.e. $g\left(\lambda Z^{\alpha}\right)=g\left(Z^{\alpha}\right)$ for all $\lambda \neq 0$;

(ii) $\operatorname{det}(g)=1$;

(iii) $g(Z)^{*}=g\left(Z^{*}\right)$, where the $*$ on the left-hand side denotes complex conjugate transpose;

(iv) $g\left(Z^{\alpha}\right)$ is complex-analytic in a suitable region (by "suitable", it is meant that $g\left(x^{P Q} \pi_{Q}, \pi_{R}\right)$ is analytic for $(x, \zeta) \in \mathscr{R} \times(U \cap \hat{U})$, where $\mathscr{R}$ is some region in $\mathbb{R}^{4}$; this makes sense because $g$, for a fixed value of $x$, is homogeneous of degree zero in $\pi_{P}$ by condition (i), and so is a function of $\zeta=\pi_{1} / \pi_{2}$ ); 
(v) g can be "split":

$$
g(x \cdot \pi, \pi)=\hat{h}(x, \zeta) h(x, \zeta)^{-1},
$$

where $\hat{h}$ and $h$ are 2 -by-2 matrices which are analytic on $\mathscr{R} \times \hat{U}$ and $\mathscr{R} \times U$ respectively.

Let $D_{P}$ denote the differential operator $D_{P}=\partial_{P 1}-\zeta \partial_{P 2}$, where $\partial_{P Q}=\partial / \partial x^{P Q}$. Then $D_{P}\left(x^{Q R} \pi_{R}\right)=0$, so operating on equation (3) with $D_{P}$ yields

$$
\hat{h}^{-1} D_{P} \hat{h}=h^{-1} D_{P} h .
$$

Now the left-hand side of this equation is analytic on $\mathscr{R} \times \hat{U}$ and the righthand side on $\mathscr{R} \times U$, so both sides must be analytic on $\mathscr{R} \times \mathbb{C I P}^{1}$, whence (by a generalized form of Liouville's theorem), both sides must be linear in $\zeta$. This enables us to define functions $A_{P Q}(x)$ by

$$
A_{P 1}-\zeta A_{P 2}=-i h^{-1} D_{P} h .
$$

This field $A_{P Q}(x)$ (or, equivalently, $A_{\mu}(x)$ defined by $A_{\mu} d x^{\mu} \equiv A_{P Q} d x^{P Q}$ ) is the gauge potential.

To see that $A_{\mu}$ satisfies the self-duality equations is straightforward. Operating on (4) with $\varepsilon^{P Q} D_{Q}$ (where $\varepsilon^{00}=\varepsilon^{11}=0, \varepsilon^{01}=-\varepsilon^{10}=1$ ) gives equations on $A_{P Q}$ which (translated into the $x^{\mu}$-coordinates) are

$$
\begin{gathered}
\frac{1}{2} \varepsilon_{\mu \nu \alpha \beta} G_{\alpha \beta}=G_{\mu \nu}, \\
G_{\mu \nu} \equiv \partial_{\mu} A_{\nu}-\partial_{\nu} A_{\mu}+i\left[A_{\mu}, A_{\nu}\right] .
\end{gathered}
$$

Certainly $A_{\mu}(x)$ is smooth in $\mathscr{R}$ (in fact, real-analytic), because by assumption the matrix $h$ is. Finally, we would like to be sure that $A_{\mu}$ takes values in the Lie algebra of $S U(2)$, i.e. that trace $\left(A_{\mu}\right)=0$ and $A_{\mu}^{*}=A_{\mu}$. This requires a discussion of the gauge freedom involved in the above construction, which arises as follows.

The splitting (3) is not unique: the freedom in $h$ and $\hat{h}$ is $h \mapsto h \Lambda, \hat{h} \mapsto \hat{h} \Lambda$, where $\Lambda$ is a 2-by-2 non-singular matrix of complex-valued functions of $x$; in other words, $\Lambda(x) \in G L(2, \mathbb{C})$. This leads to

$$
A_{\mu} \mapsto \Lambda^{-1} A_{\mu} \Lambda-i \Lambda^{-1} \partial_{\mu} \Lambda,
$$

a $G L(2, \mathbb{C})$ gauge transformation of $A_{\mu}$. As a consequence of conditions (ii) and (iii) on the matrix $g$, it is possible to choose a gauge such that trace $\left(A_{\mu}\right)=0$ and $A_{\mu}^{*}=A_{\mu}$. This is proved in [7]; the idea is that $h$ and $\hat{h}$ can be chosen to satisfy $\hat{h}\left(x,-\bar{\zeta}^{-1}\right)= \pm h(x, \zeta)^{*-1}$, from which the above conditions on $A_{\mu}$ follow.

We see, therefore, that a matrix $g$ satisfying requirements (i)-(v) produces a smooth solution $A_{\mu}$ of the $S U(2)$ self-duality equations on the region $\mathscr{R}$ in $\mathbb{R}^{4}$. In fact, this procedure produces all such solutions [13], but this will not be proved here.

Finally, it should be noted that there is some freedom in the matrix $g$ : many different $g$ 's will produce the same $A_{\mu}$. This may be thought of 
geometrically if we recognize that $g$ is the "patching matrix" of a vector bundle over twistor space, and there is an equivalence relation on the set of all such vector bundles [13]. What it amounts to is the following:

$$
g \mapsto \tilde{g}=\hat{m} g m,
$$

where $\hat{m}$ and $m$ are non-singular 2-by-2 matrices of functions of $Z^{\alpha}$, homogeneous of degree zero, such that $\hat{m}(x \cdot \pi, \pi)$ and $m(x \cdot \pi, \pi)$ are analytic for $(x, \zeta) \in \mathscr{R} \times \hat{U}$ and $(x, \zeta) \in \mathscr{R} \times U$ respectively. We say that $g$ and $\tilde{g}$ are equivalent. It is easy to see that $g$ and $\tilde{g}$ produce the same $A_{\mu}$ (essentially, this is because $m$ and $\hat{m}$ depend on $x$ only through the combination $x^{P Q} \pi_{Q}$, which is annihilated by $D_{P}$ ).

\section{The Ansatz $\mathscr{A}_{n}$}

The difficult part of the above solution procedure is that of finding the matrices $h$ and $\hat{h}$. Even if we know (by some implicit argument) that a splitting exists, it may be impossible in practice to find explicit expressions for $h$ and $\hat{h}$. There is, however, a large class of cases that can be treated explicitly, namely those arising from matrices of the form

$$
\tilde{g}=\left[\begin{array}{cc}
\zeta^{n} e^{f} & \Gamma \\
0 & \zeta^{-n} e^{-f}
\end{array}\right],
$$

where $f$ and $\Gamma$ are functions of $Z^{\alpha}$, homogeneous of degree zero and analytic for $(x, \zeta) \in \mathscr{R} \times(U \cap \hat{U})$. The reason that this matrix is denoted $\tilde{g}$ rather than $g$ is not hard to see: if we required it to be subject to the reality condition $g(Z)^{*}$ $=g\left(Z^{*}\right)$, then $\Gamma$ would have to vanish. So instead we require that $\tilde{g}$ be equivalent to a matrix $g$ satisfying the reality condition. We shall return to this question of reality later; first let us consider how to obtain $A_{\mu}$ from $\tilde{g}$, using an argument which generalizes that in [11].

From now on, let the number $n$ which appears in $\tilde{g}$ be a positive integer (the $n \leqq 0$ cases are essentially trivial). The first step in splitting $\tilde{g}$ is to split the function $f$ :

$$
f(x \cdot \pi, \pi)=\hat{\mu}(x, \zeta)-\mu(x, \zeta)
$$

where $\mu$ and $\hat{\mu}$ are analytic functions on $\mathscr{R} \times U$ and $\mathscr{R} \times \hat{U}$ respectively. That such a splitting of $f$ is always possible is a consequence of elementary complex analysis; in fact, we can express $\mu$ and $\hat{\mu}$ as contour integrals

$$
\begin{aligned}
& \mu\left(x, \zeta^{\prime}\right)=\oint \Omega \\
& \hat{\mu}\left(x, \zeta^{\prime}\right)=\oint \Omega,
\end{aligned}
$$

where $\Omega=(2 \pi i)^{-1} f\left(x^{P Q} \pi_{Q}, \pi_{R}\right) d \zeta\left(\zeta-\zeta^{\prime}\right)^{-1}$, and the contour of integration is $|\zeta|$ $=1, \oint(2 \pi i \zeta)^{-1} d \zeta=1$. The difference between $\mu$ and $\hat{\mu}$ arises from the position of $\zeta^{\prime}$ in relation to the contour: for $\mu$, take $\left|\zeta^{\prime}\right|<1$; and for $\hat{\mu}$ take $\left|\zeta^{\prime}\right|>1$. Equation (7) then follows from Cauchy's integral formula. 
If we now multiply the matrix $\tilde{g}$ on the left by the matrix $\operatorname{diag}\left(e^{-\hat{\mu}}, e^{\hat{\mu}}\right)$ and on the right by $\operatorname{diag}\left(e^{\mu}, e^{-\mu}\right)$, we obtain

$$
\left[\begin{array}{cc}
\zeta^{n} & \rho(x, \zeta) \\
0 & \zeta^{-n}
\end{array}\right]
$$

where $\rho=\Gamma e^{-\mu-\hat{\mu}}$; and Corrigan et al [11] have already shown how to split a matrix of this form into matrices $\hat{h}$ and $h$ (which they denote $h$ and $k$ respectively). The present case is more general than theirs, because in their case the function $\rho$ depended on $x$ only through the combination $x^{P Q} \pi_{Q}$, whereas here its dependence is more general (since that of $\mu$ and $\hat{\mu}$ is more general). But this difference does not affect the computation of $h$ and $\hat{h}$; it only enters at the final stage, when $A_{\mu}$ is computed. Choosing Yang's $R$-gauge [14], one obtains the following (for more details, see the appendix).

Acting on Eq. (7) with $D_{P}$ gives $D_{P} \mu=D_{P} \hat{\mu}$, which (again using a Liouvilletype argument) enables us to define a field $B_{P Q}(x)$ by

$$
B_{P 1}-\zeta B_{P 2}=D_{P} \mu \text {. }
$$

In fact, $B_{\mu}$ is the potential for a self-dual Maxwell field, and the transformation $f \rightarrow(\mu, \hat{\mu}) \rightarrow B_{\mu} \rightarrow$ Maxwell field is exactly the Penrose transform [15]. Now define fields $\Delta_{r}(x)$ for $r=0, \pm 1, \pm 2, \ldots$ by

$$
\Delta_{r}(x)=(2 \pi i)^{-1} \oint \zeta^{r-1} \rho(x, \zeta) d \zeta,
$$

where the contour is the same as the one used previously. It is easily checked that the $\Delta_{r}$ satisfy

$$
\left(\partial_{P 1}+2 B_{P 1}\right) \Delta_{r}=\left(\partial_{P 2}+2 B_{P 2}\right) \Delta_{r+1} .
$$

Let $M$ be the $n$-by- $n$ matrix defined by $M_{r s}=\Delta_{r+s-n-1}$ for $1 \leqq r, s \leqq n$ (Corrigan et al [11] refer to this matrix as $D^{(n)}$ ), and let $E, F$ and $G$ be the "corner elements" of its inverse: $E=\left(M^{-1}\right)_{11}, F=\left(M^{-1}\right)_{1 n}, G=\left(M^{-1}\right)_{n n}$. Then the gauge potential $A_{\mu}$ is given by

$$
\begin{aligned}
A_{P 1} & =\frac{-i}{2 F}\left[\begin{array}{cc}
\nabla_{P 1} F & 0 \\
-2 \nabla_{P 2} G & -\nabla_{P 1} F
\end{array}\right], \\
A_{P 2} & =\frac{-i}{2 F}\left[\begin{array}{cc}
-\nabla_{P 2} F & -2 \nabla_{P 1} E \\
0 & \nabla_{P 2} F
\end{array}\right],
\end{aligned}
$$

where $\nabla_{P Q}=\partial_{P Q}-2 B_{P Q}$.

This, then, is the ansatz $\mathscr{A}_{n}$ : given the functions $f$ and $\Gamma$ which determine $\tilde{g}$, the gauge potential (11) obtained from them is a solution of the self-duality equations. It generalizes the ansatz $\mathscr{A}_{n}$ of $[10,11]$, and coincides with it if $f=0$. Since $f$ and $\Gamma$ are effectively functions of three variables (being homogeneous of degree zero), $\mathscr{A}_{n}$ provides solutions of the self-duality equations depending on two free functions of three variables each.

An important question remains to be answered: what are the conditions on $f$ and $\Gamma$ which ensure that $A_{\mu}$ is smooth and $s u(2)$-valued? This is a com- 
plicated problem, and all that will be done here is to give some sufficient conditions on $f$ and $\Gamma$. First, let us deal with smoothness. It is proved in the appendix that if the fields $\Delta_{r}$, for $|r| \leqq n$, are smooth in the region $\mathscr{R}$ in $\mathbb{R}^{4}$, and if $\operatorname{det}(M)$ is nowhere-vanishing in $\mathscr{R}$, then $A_{\mu}$ is real-analytic in $\mathscr{R}$. This condition on the $\Delta_{r}$ is not necessary for smoothness of $A_{\mu}$, merely sufficient.

Secondly, we come to the question of reality, which was mentioned at the beginning of this section. The matrix $\tilde{g}$ has to be equivalent to a real matrix $g$, i.e. one satisfying $g(Z)^{*}=g\left(Z^{*}\right)$. What will be done here is to give two sets of conditions on $f$ and $\Gamma$, each of which is sufficient to ensure reality.

Case (a). $f$ imaginary, $\Gamma$ real, $n$ odd. By this it is meant that $f$ and $\Gamma$ satisfy $\overline{f(Z)}=-f\left(Z^{*}\right)$ and $\overline{\Gamma(Z)}=\Gamma\left(Z^{*}\right)$. These conditions clearly imply that the matrix

$$
g=\left[\begin{array}{cc}
\zeta^{n} e^{f} & \Gamma \\
0 & \zeta^{-n} e^{-f}
\end{array}\right]\left[\begin{array}{cr}
0 & -1 \\
1 & 0
\end{array}\right]=\left[\begin{array}{cc}
\Gamma & -\zeta^{n} e^{f} \\
\zeta^{-n} e^{-f} & 0
\end{array}\right]
$$

satisfies the reality condition $g(Z)^{*}=g\left(Z^{*}\right)$, provided that the integer $n$ is odd. (Recall that $*$ maps $\zeta$ to $-\bar{\zeta}^{-1}$.) If $n=1$ and $f=0$, we get the well-known Corrigan-Fairlie-'t Hooft-Wilczek ansatz.

Case (b). $f$ real, $\Gamma=H^{-1}\left[e^{f}+(-1)^{n} e^{-f}\right]$, where $H=\left(\pi_{1} \pi_{2}\right)^{-n} P(Z)$ and $P\left(Z^{\alpha}\right)$ is a homogeneous polynomial of degree $2 n$, satisfying $\overline{P(Z)}=(-1)^{n} P\left(Z^{*}\right)$. There is no restriction on $n$. Then the matrix

$$
g=\left[\begin{array}{cc}
\zeta^{n} e^{f} & \Gamma \\
0 & \zeta^{-n} e^{-f}
\end{array}\right]\left[\begin{array}{cc}
0 & -1 \\
1 & \zeta^{n} H
\end{array}\right]=\left[\begin{array}{cc}
\Gamma & (-1) \zeta^{n} e^{-f} \\
\zeta^{-n} e^{-f} & H e^{-f}
\end{array}\right]
$$

is real, as is easily checked. Note that $\zeta^{n} H(x \cdot \pi, \pi)=\left(\pi_{2}\right)^{-2 n} P(x \cdot \pi, \pi)$ is necessarily analytic for $\zeta \in U$ (since $\pi_{2}=0$ occurs in $\hat{U}$ ), as required by the definition of equivalence given in Sect. 2. The multi-monopole solutions are generated by $f$ 's and $\Gamma$ 's belonging to this class, as we shall see in the next section.

Finally, it should be emphasized that in order to get a real gauge field, it is not necessary for $f$ and $\Gamma$ to belong to either class (a) or class (b); it is merely sufficient.

\section{The 2-Monopole Solution}

If we assume that $A_{\mu}$ is independent of the "imaginary time" coordinate $x^{0}$, then the self-duality equations (5) become the Yang-Mills-Higgs-Bogomolny equations

$$
\begin{aligned}
G_{j k} & =-\varepsilon_{j k \ell} D_{\ell} \phi, \\
G_{j k} & =\partial_{j} A_{k}-\partial_{k} A_{j}+i\left[A_{j}, A_{k}\right], \\
D_{j} \phi & =\partial_{j} \phi+i\left[A_{j}, \phi\right],
\end{aligned}
$$

where the $x^{0}$-component $A_{0}$ of the gauge potential has been identified as the Higgs field $\phi[16,6,7]$. These are the equations for minimum-energy, static, 
purely magnetic Yang-Mills-Higgs fields, in the Prasad-Sommerfield limit where the Higgs self-interaction vanishes $[5,6]$. The appropriate boundary condition is

$$
\|\phi\| \rightarrow 1 \quad \text { as } r \rightarrow \infty,
$$

where $\|\phi\|^{2}=-\operatorname{det}(\phi)$ and $r^{2}=x^{2}+y^{2}+z^{2}$ (from now on we shall use the coordinates $\left.x=x^{3}, y=x^{1}, z=x^{2}\right)$. Smooth $s u(2)$-valued solutions of the $Y M H B$ equations, with this boundary condition, are called multi-monopoles.

The norm $\|\phi\|$ of $\phi$ necessarily has the form $\|\phi\|=1-\frac{1}{2} n / r+O\left(r^{-2}\right)$ as $r \rightarrow \infty$, where $n$ is a positive integer called the topological charge [17]. By definition, we say that the location of the monopole(s) is at the point(s) where $\phi$ vanishes.

The first monopole solution to be discovered was spherically symmetric and had $n=1$ [5]. This solution can be seen to arise from the ansatz $\mathscr{A}_{1}$ [16], although that was not how it was first found. Then it was observed that $\mathscr{A}_{2}$ generates an axially-symmetric $n=2$ solution [7]. And it seems likely that $\mathscr{A}_{n}$, for $n \geqq 3$, generates an axially-symmetric solution of charge $n$ (at the time of going to press, there is still a slight gap in the proof of this) [8]. All these monopoles are located at precisely one point in space; this is a consequence of the axial symmetry [18].

Meanwhile, Taubes had given an existence theorem for non-axially-symmetric multi-monopole solutions located at several points [6]. But his proof is only valid if the monopoles are sufficiently far apart. It seemed likely that solutions representing monopoles close together also existed, and this is indeed the case: it was recently announced that the ansatz $\mathscr{A}_{2}$ generates such a 2 monopole solution [12]. The details of this construction will now be given.

From the analysis of Sect. 2, it is easy to see that if $g$ depends on $\omega^{1}$ and $\omega^{2}$ only through the combination

$$
\gamma=-i \omega^{1} / \pi_{1}+i \omega^{2} / \pi_{2}
$$

then $g(x \cdot \pi, \pi)$ is independent of the coordinate $x^{0}$. Substituting $\omega=x \cdot \pi$ into $\gamma$ gives

$$
\gamma(x, y, z, \zeta)=\xi \zeta-2 z-\bar{\xi} \zeta^{-1},
$$

where $\xi=x+i y$. Notice that $\gamma$ is real, in the sense that $\overline{\gamma(x, y, z, \zeta)}=\gamma(x, y, z$, $\left.-\bar{\zeta}^{-1}\right)$. So from now on we require that $g$ be a function of $\gamma$ and $\zeta$ only.

Let us proceed to apply the results of the previous section, and to use the ansatz $\mathscr{A}_{2}$ with the reality condition of case (b). The function $H$ has to be a "real" polynomial of degree two in $\gamma, \zeta$ and $\zeta^{-1}$. The most general such polynomial contains nine real parameters. One of these is an overall scale factor and is irrelevant: it drops out when one finally computes $A_{\mu}$. Six of the others may be removed by making a rigid motion of the $x y z$-space. This leaves us with two parameters, and an $H$ of the form

$$
\begin{aligned}
H & =\gamma^{2}+\delta, \\
\delta & =\frac{1}{2} p q\left(\zeta-\zeta^{-1}\right)+q,
\end{aligned}
$$

$p$ and $q$ being real parameters. 
Now we have to select the function $f$. We want $\Gamma$ to be smooth even when its denominator $H$ vanishes (this will guarantee that the $\Delta_{r}$ are smooth), and we want $f$ to be linear in $\gamma$ (this seems to be required by the boundary condition on $\phi$. The simplest $f$ having these properties is $f=\frac{1}{2} \pi \delta^{-\frac{1}{2}} \gamma$. (From now on, $\pi$ denotes the usual real constant, and has nothing to do with the variables $\pi_{p}$.) Let us assume that $q>0$, and choose the branch of $\delta^{\frac{1}{2}}$ which has positive real part; then $f$ is analytic in a neighborhood of $|\zeta|=1$, as required.

Now that we have $f$ and $\Gamma$, we can proceed with the calculation. The first step is to split $f$ into $\hat{\mu}-\mu$. Here a choice arises, since $\mu$ and $\hat{\mu}$ are not unique: the contour integral formula (8) for $\mu$ and $\hat{\mu}$ is just one solution. For there is the freedom $\mu \mapsto \mu+\lambda, \hat{\mu} \mapsto \hat{\mu}+\lambda$, where $\lambda=\lambda\left(x^{P Q}\right)$; this corresponds to a gauge transformation of the Maxwell potential $B_{\mu}$. And if $f$ is linear in $\omega^{P}$ (as it is in our case), then $B_{\mu}$ can be gauged away altogether, at the cost of introducing explicit $x^{0}$-dependence in the intermediate stages of the calculation. Since $B_{\mu}=0$ makes things simpler, we shall choose this gauge. But it should be emphasized that it is possible to do the calculation (and obtain the same final answer for the Yang-Mills field $A_{\mu}$ ) without the $x^{0}$-variable ever making an appearance.

So, suitably adapting the contour integral formula, we find that $\mu\left(x^{P Q}, \zeta\right)$ and $\hat{\mu}\left(x^{P Q}, \zeta\right)$ are given by

$$
\frac{1}{4} q^{-\frac{1}{2}} \int_{0}^{2 \pi}(1+i p \sin \theta)^{-\frac{1}{2}} \Lambda d \theta,
$$

where $\Lambda=-i t+\left(\zeta-e^{i \theta}\right)^{-1}\left(\bar{\xi}+z \zeta-e^{i \theta} \xi \zeta+e^{i \theta} z\right)$, and the square root in the integrand is taken to have positive real part. For $|\zeta|<1,(14)$ gives $\mu$; and for $|\zeta|>1,(14)$ gives $\hat{\mu}$. It is easily checked that $D_{P} \mu=0$, so $B_{\mu}=0$ as required.

The next step is that of computing the functions $\Delta_{r}$ according to the integral formula (9). This integral can be done by finding the zeros of $H$ and computing the residues of $\rho$ at each one, but since $H$ is effectively a quartic polynomial in $\zeta$, this is messy. And of course the integral (14) is also rather messy. For the time being, let us just make the observation that because of the careful way in which we chose $f$, the function $\rho\left(x^{P Q}, \zeta\right)$ is analytic in a neighbourhood of $|\zeta|=1$, for all $x^{P Q}$; and so the functions $\Delta_{r}(x)$ are everywhere real-analytic.

It is possible to find out what happens as $r \rightarrow \infty$, for then $H$ can be "approximately" factorized and the integral (9) can be computed. This calculation gives

$$
\begin{aligned}
\operatorname{det}(M) & \equiv \Delta_{1} \Delta_{-1}-\Delta_{0}^{2}=\left\{-\frac{1}{16} r^{-4}+O\left(r^{-5}\right)\right\} \exp \{4 I(i t+r)\} \\
\|\phi\| & =I+O\left(r^{-1}\right),
\end{aligned}
$$

where $I=\frac{1}{4} q^{-\frac{1}{2}} \int_{0}^{2 \pi}(1+i p \sin \theta)^{-\frac{1}{2}} d \theta$.

So in order to get the right boundary behaviour we must have $I=1$, and this determines the constant $q$ in terms of $p$. We see also that $e^{-4 i t} \operatorname{det}(M) \rightarrow-\infty$ as $r \rightarrow \infty$. To be certain that the gauge field $A_{\mu}$ defined by (11) is non-singular, 
it is sufficient to prove that $\operatorname{det}(M)$ is nowhere zero (since we already know that the $\Delta_{r}$ are smooth). Certainly $\operatorname{det}(M) \neq 0$ for $p$ sufficiently small, as the following argument shows.

When $p=0$, the field becomes that of the axisymmetric 2-monopole solution [7], where the calculations are relatively simple. In particular,

$$
\left.e^{-4 i t} \operatorname{det}(M)\right|_{p=0}=\frac{1}{4}\left(a^{2}+b^{2}\right)^{-2}\left\{\left(c^{2}-b^{2}\right)^{-1} \cos ^{2}(2 b)-\left(a^{2}+c^{2}\right)^{-1} \cosh ^{2}(2 a)\right\},
$$

where $c=\pi / 4$ and $(a+i b)^{2}=(z+i c)^{2}+\xi \bar{\xi}$. Some simple estimates show that the expression (15) is negative for all $x, y, z$; and, in fact, it is bounded away from zero by a finite amount. Therefore, since $\operatorname{det}(M)$ is analytic in $p$ and $e^{-4 i t} \operatorname{det}(M) \rightarrow-\infty$ as $r \rightarrow \infty$, there exists a positive number $p_{0}$ such that $\operatorname{det}(M) \neq 0$ for all $x, y, z$ and all $|p|<p_{0}$.

The conclusion is, therefore, that for $|p|<p_{0}$ our construction yields a monopole solution. Its topological charge is $n=2$ (by continuity, since the $p=0$ monopole has $n=2$ [7]. The claim is that (at least for sufficiently small $p$ ) the solution for $p \neq 0$ represents two monopoles located at two distinct points in space, and that the distance between them is related to $p$. Consequently, the solution is not symmetric about any axis in space, even the line joining the two points [18], except of course when $p=0$. To see that this claim is valid, it suffices to compute the Higgs field $\phi$ for infinitesimal values of $x, y, z$ and $p$. (In the $p=0$ case, $\phi$ vanishes at $x=y=z=0$.) This infinitesimal calculation is easily carried out, and yields $\left(\phi=\phi^{a} \sigma^{a}\right.$, where $\sigma^{a}$ are the Pauli matrices):

$$
\begin{aligned}
& \phi^{1}=\left(32 \lambda^{2}\right)^{-1}\left(p^{2} \lambda^{2}-x^{2}+y^{2}\right), \\
& \phi^{2}=-\left(16 \lambda^{2}\right)^{-1} x y, \\
& \phi^{3}=\left(8 / \pi^{2}-1\right)(2 z-p x),
\end{aligned}
$$

plus third-and higher-order terms, where $\lambda^{-2}=32\left(12 / \pi^{2}-1\right)$. We see that the zeros of $\phi$ are located at $x= \pm p \lambda, y=0, z= \pm \frac{1}{2} p^{2} \lambda$; so as $p$ moves away from 0 , the superimposed 2-monopole separates into two distinct monopoles.

\section{Conclusion}

We have seen that there is a sequence $\mathscr{A}_{1}, \mathscr{A}_{2}, \mathscr{A}_{3}, \ldots$ of ansätze, each one of which generates a complex-valued solution of the self-duality equations (5) depending on two arbitrary complex-analytic functions of three variables each. One expects that the general complex self-dual field would depend on three arbitrary functions of three variables each. Is it the case that the union of all the ansätze produces all self-dual solutions, or perhaps a dense subset of the space of all solutions? It is conceivable that this may be the case (there is enough "room" in the countable sequence of ansätze), but this remains a conjecture.

What one can say is that the ansätze are certainly relevant to the two most important cases where the self-duality equations are used - instantons and monopoles. For it is a theorem that all self-dual instantons can be obtained 
out of the union of the ansätze [10]. And while no corresponding theorem for monopoles is known at present, it does appear that the ansätze will produce, for any $n>0$, solutions of charge $n$ possessing $(4 n-1)$ degrees of freedom [19], which could well be the most general monopole solutions [20].

It seems likely that the expressions for general $n$-monopole solutions, as functions of $x, y$ and $z$ are so complicated that there would be little point in trying to write them out. Of course, since we have explicit formulae, the fields could be computed numerically to any desired degree of accuracy. One attraction of the technique presented here is that the matrices $g$ are relatively simple, even when the corresponding space-time fields $A_{\mu}$ are extremely complicated. So one can deduce much about instantons and monopoles (such as their existence!) without having to write down space-time expressions for them.

\section{Appendix}

The purpose of this appendix is to add some details to the discussion of Sect. 3, and to prove that if the $\Delta_{r}$ are smooth and $\operatorname{det}(M) \neq 0$ (where $M_{r s}=\Delta_{r+s-n-1}$ is an $n$-by- $n$ matrix), then $A_{\mu}$ is smooth in some gauge. The analysis here is analogous to that in [11].

We want functions $\hat{a}, \hat{b}, \hat{c}, \hat{d}, a, b, c, d$ of $x$ and $\zeta$, with the first four analytic in $\mathscr{R} \times \hat{U}$ and the last four in $\mathscr{R} \times U$, such that

$$
\tilde{g}=\left[\begin{array}{cc}
\zeta^{n} e^{f} & \Gamma \\
0 & \zeta^{-n} e^{-f}
\end{array}\right]=\left[\begin{array}{ll}
\hat{a} & \hat{b} \\
\hat{c} & \hat{d}
\end{array}\right]\left[\begin{array}{rr}
d & -b \\
-c & a
\end{array}\right]
$$

and $a d-b c=\hat{a} \hat{d}-\hat{b} \hat{c}=1$. This is the "split" of $\tilde{g}$ into $\hat{h}$ and $h$. The most general form for these eight functions is as follows. It involves $\mu, \hat{\mu}$ and $\Delta_{r}$ (recall that $f=\hat{\mu}-\mu$ and $\rho=\Gamma e^{-\mu-\hat{\mu}}=\sum_{-\infty}^{\infty} \Delta_{-k} \xi^{k}$ ), and $2 n+2$ complex-valued functions of $x$, namely $c_{0}, \ldots, c_{n}, d_{0}, \ldots, d_{n}$.

$$
\begin{aligned}
e^{\mu} c & =e^{\hat{\mu}} \zeta^{n} \hat{c}=\sum_{k=0}^{n} c_{k} \zeta^{k}, \\
e^{\mu} d & =e^{\hat{\mu}} \zeta^{n} \hat{d}=\sum_{k=0}^{n} d_{k} \zeta^{k}, \\
\hat{a} & =e^{\hat{\mu}} \sum_{k=-\infty}^{0} \theta_{k} \zeta^{k}, \\
\hat{b} & =e^{\hat{\mu}} \sum_{k=-\infty}^{0} \phi_{k} \zeta^{k}, \\
a & =-e^{\mu} \sum_{k=1}^{\infty} \theta_{k} \zeta^{k-n}, \\
b & =-e^{\mu} \sum_{k=1}^{\infty} \phi_{k} \zeta^{k-n},
\end{aligned}
$$


where

$$
\begin{aligned}
\theta_{k} & =\sum_{l=0}^{n} \Delta_{\ell-k} c_{\ell}, \\
\phi_{k} & =\sum_{\ell=0}^{n} \Delta_{\ell-k} d_{\ell} .
\end{aligned}
$$

In order that $a$ and $b$ should be analytic at $\zeta=0$, it is necessary to constrain $c_{k}$ and $d_{k}$ in such a way that

$$
\theta_{k}=\phi_{k}=0 \quad \text { for } 1<k<n \text {. }
$$

And the unimodularity contition $a d-b c=1$ amounts to

$$
c_{0} \phi_{n}-d_{0} \theta_{n}=1 \text {. }
$$

The Eqs. (16) and (17) give $2 n-1$ conditions on the $2 n+2$ functions $c_{k}, d_{k}$; this leaves, as one would expect, three free functions of $x$ representing the gauge freedom. But we need to be sure that the Eqs. (16) and (17) can in fact be solved, and the condition which ensures this is $\operatorname{det}(M) \neq 0$, as we shall now see.

Consider the $(n+1)$-by- $(n+1)$ matrix

$$
N=\left[\begin{array}{cccc}
1 & 0 & \ldots & 0 \\
\Delta_{-n} & \Delta_{-n+1} & \ldots & \Delta_{0} \\
\vdots & \vdots & & \vdots \\
\Delta_{-1} & \Delta_{0} & \ldots & \Delta_{n-1}
\end{array}\right] .
$$

Clearly $\operatorname{det}(N)=\operatorname{det}(M)$, so if $\operatorname{det}(M) \neq 0$, then the rows $\boldsymbol{v}_{0}, \boldsymbol{v}_{1}, \ldots, \boldsymbol{v}_{n}$ of $N$ are linearly independent. We think of $\boldsymbol{v}_{k}$ as vectors in $\mathbb{C}^{n+1}$, and also of $\mathbf{c}=\left(c_{0}, \ldots, c_{n}\right)$ and $\mathbf{d}=\left(d_{0}, \ldots, d_{n}\right)$ as such vectors. Eq. (16) says that $\mathbf{c}$ and $\mathbf{d}$ are orthogonal to $v_{2}, \ldots, v_{n}$ (with respect to the "complex-Euclidean" metric on $\left.\mathbb{C}^{n+1}\right)$. In other words, $\mathbf{c}$ and $\mathbf{d}$ lie in the complex 2-plane $P$ orthogonal to $\boldsymbol{v}_{2}, \ldots, \boldsymbol{v}_{n}$.

$\operatorname{Det}(N) \neq 0$ also implies that the projections $\omega_{0}$ and $\omega_{1}$ of $\boldsymbol{v}_{0}$ and $\boldsymbol{v}_{1}$ into $P$ are linearly independent. It follows that Eq. (17), which can be written

$$
\left(\omega_{0} \cdot \mathbf{c}\right)\left(\omega_{1} \cdot \mathbf{d}\right)-\left(\omega_{0} \cdot \mathbf{d}\right)\left(\omega_{1} \cdot \mathbf{c}\right)=1
$$

has a solution (in fact, many solutions) for $\mathbf{c}$ and $\mathbf{d}$. This proves the desired result, for then $\tilde{g}$ can be split as in Eq. (3).

Sect. 3 we chose Yang's $R$-gauge [14] in arriving at Eq. (11). This gauge corresponds to $d_{0}=c_{n}=0, c_{0}=d_{n}$. A priori, this might be a singular gauge, for the function $F$ may vanish without $\operatorname{det}(M)$ vanishing. In view of what we proved above, such a singularity can always be removed by a gauge transformation.

\section{References}

1. Belavin, A.A., Polyakov, A.M., Schwarz, A.S., Tyupkin, Yu.S.: Phys. Lett. B59, 85-87 (1975)

2. Jackiw, R., Nohl, C., Rebbi, C.: Phys. Rev. D15, 1642-1646 (1977) 
3. Atiyah, M.F., Hitchin, N.J., Drinfeld, V.G., Manin, Yu.I.: Phys. Lett. A65, 185-187 (1978)

4. 't Hooft, G.: Nucl. Phys. B 79, 276-284 (1974)

5. Prasad, M.K., Sommerfield, C.M.: Phys. Rev. Lett. 35, 760-762 (1975)

6. Jaffe, A., Taubes, C.: Vortices and monopoles. Boston: Birkhäuser, 1980

7. Ward, R.S.: Commun. Math. Phys. 79, 317-325 (1981)

8. Prasad, M.K.: Commun. Math. Phys. 80, 137-149 (1981)

9. Ward, R.S.: Phys. Lett. A61, 81-82 (1977)

10. Atiyah, M.F., Ward, R.S.: Commun. Math. Phys. 55, 117-124 (1977)

11. Corrigan, E.F., Fairlie, D.B., Yates, R.G., Goddard, P.: Commun. Math. Phys. 58, 223-240 (1978)

12. Ward, R.S.: Phys. Lett. B, (to appear)

13. Atiyah, M.F.: Geometry of Yang-Mills Fields. Pisa: Scuola Normale Superiore 1979

14. Yang, C.N.: Phys. Rev. Lett. 38, 1377-1379 (1977)

15. Eastwood, M.G., Penrose, R., Wells, R.O., Jr.: Commun. Math. Phys. 78, 305-351 (1981)

16. Manton, N.S.: Nucl. Phys. B 135, 319-332 (1978)

17. Arafune, J., Freund, P.G.O., Goebel, C.J.: J. Math. Phys. (NY) 16, 433-437 (1975)

18. Houston, P., O'Raifeartaigh, L.: On monopole systems with weak axial symmetry. Preprint, Dublin

19. Corrigan, E., Goddard, P.: Commun. Math. Phys. 80, 575-587 (1981)

20. Weinberg, E.: Phys. Rev. D 20, 936-944 (1979)

Communicated by A. Jaffe

Received April 8, 1981

Note Added in Proof. N. Hitchin has recently shown that every $n$-monopole solution can be obtained from the ansatz $\mathscr{A}_{n}$, with $f=\gamma$. This makes it even more likely that the proposed $(4 n-1)$ parameter family of solutions [19] is correct, and represents the general multi-monopole configuration. In [19]. the function $f$ (which there is denoted $\theta$ ) is a more general polynomial in $\gamma$; but it can be reduced to $f=\gamma$ by an equivalence transformation on $g$ and a coordinate transformation in $\mathbb{R}^{3}$. The same remark applies to the function $f=\frac{1}{2} \pi \delta^{-\frac{1}{2}} \gamma$ used in Sect. 4 above. 